\title{
Higher spherical algebras
}

\author{
Karin ERdmann AND ANDrZej Skowroński(D)
}

\section{Dedicated to Ibrahim Assem on the occasion of his 70th birthday.}

\begin{abstract}
We introduce and study higher spherical algebras, an exotic family of finite-dimensional algebras over an algebraically closed field. We prove that every such an algebra is derived equivalent to a higher tetrahedral algebra studied in Erdmann and Skowroński (Algebras Represent Theory $22: 387-406,2019)$, and hence that it is a tame symmetric periodic algebra of period 4 .
\end{abstract}

Mathematics Subject Classification. 16D50, 16E30, 16G60, 18E30.

Keywords. Periodic algebra, Symmetric algebra, Tame algebra, Higher tetrahedral algebra, Higher spherical algebra, Derived equivalence.

1. Introduction and main results. Throughout this paper, $K$ will denote a fixed algebraically closed field. By an algebra, we mean an associative finitedimensional $K$-algebra with an identity. For an algebra $A$, we denote by $\bmod A$ the category of finite-dimensional right $A$-modules and by $D$ the standard duality $\operatorname{Hom}_{K}(-, K)$ on $\bmod A$. An algebra $A$ is called self-injective if $A_{A}$ is injective in $\bmod A$, or equivalently, the projective modules in $\bmod A$ are injective. A prominent class of self-injective algebras is formed by the symmetric algebras $A$ for which there exists an associative, non-degenerate symmetric $K$ bilinear form $(-,-): A \times A \rightarrow K$. Classical examples of symmetric algebras are provided by the blocks of group algebras of finite groups and the Hecke algebras of finite Coxeter groups. In fact, any algebra $A$ is a quotient algebra of its trivial extension algebra $\mathrm{T}(A)=A \ltimes D(A)$, which is a symmetric algebra.

For an algebra $A$, the module category $\bmod A^{e}$ of its enveloping algebra $A^{e}=A^{\mathrm{op}} \otimes_{K} A$ is the category of finite-dimensional $A$ - $A$-bimodules. We denote by $\Omega_{A^{e}}$ the syzygy operator in $\bmod A^{e}$, which assigns to a module $M$ in $\bmod A^{e}$ the kernel $\Omega_{A^{e}}(M)$ of a minimal projective cover of $M$ in $\bmod A^{e}$. An algebra $A$ is called periodic if $\Omega_{A^{e}}^{n}(A) \cong A$ in $\bmod A^{e}$ for some $n \geq 1$, and if so, the 
minimal such $n$ is called the period of $A$. Periodic algebras are self-injective and have periodic Hochschild cohomology.

Finding or possibly classifying periodic algebras is an important and interesting problem as it has connections with group theory, topology, singularity theory, and cluster algebras. For details, we refer to the survey article [7] and the introductions of $[2,8,10]$.

We are concerned with the classification of all periodic tame symmetric algebras. Dugas [6] proved that every representation-finite self-injective algebra, without simple blocks, is a periodic algebra. The representation-infinite, indecomposable, periodic algebras of polynomial growth were classified by Białkowski et al. [2]. It is conjectured in [8, Problem] that every indecomposable symmetric periodic tame algebra of non-polynomial growth is of period 4 . There is the large class of tame symmetric algebras of period 4 , the weighted surface algebras associated to triangulations of compact real surfaces, these and their deformations are investigated in [8-11]. Weighted surface algebras contain as special cases the class of Jacobian algebras of quivers with potentials associated to orientable surfaces with punctures and empty boundary (see $[4,5,16,20]$ for details and related results). Surface triangulations have been also used to study cluster algebraic structures in Teichmüller theory $[15,17]$ and cluster algebras of topological origin [14].

Periodic algebras based on surface triangulations lead also to interesting non-periodic symmetric tame algebras. Namely, the orbit closures of periodic surface algebras in the affine varieties of associative $K$-algebra structures contain a wide class of symmetric tame algebras. They are natural generalizations of algebras of dihedral and semidihedral type, which occurred in the study of blocks of group algebras with dihedral and semidihedral defect groups (see [13] for classification of algebras of generalized dihedral type). Moreover, we get new symmetric tame algebras by taking the idempotents algebras $e \Lambda e$ of periodic surface algebras $\Lambda$. In particular, every Brauer graph algebra is of this form (see [12, Theorem 4]). Summing up, a classification of symmetric tame periodic algebras of period 4 is currently an important problem.

In this article, we introduce and study higher spherical algebras, which are "higher analogs" of the non-singular spherical algebras introduced in [11], and provide a new exotic family of tame symmetric periodic algebras of period 4 .

Let $m \geq 1$ be a natural number and $\lambda \in K^{*}$. We denote by $S(m, \lambda)$ the algebra given by the quiver $\Delta$ of the form

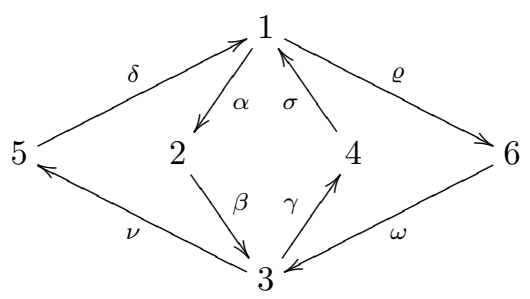


and the relations:

$$
\begin{array}{rlrl}
\beta \nu \delta & =\beta \gamma \sigma+\lambda(\beta \gamma \sigma \alpha)^{m-1} \beta \gamma \sigma, & \alpha \beta \nu & =\varrho \omega \nu, \\
\nu \delta \alpha & =\gamma \sigma \alpha+\lambda(\gamma \sigma \alpha \beta)^{m-1} \gamma \sigma \alpha, & \delta \alpha \beta & =\delta \varrho \omega, \\
\sigma \varrho \omega & =\sigma \alpha \beta+\lambda(\sigma \alpha \beta \gamma)^{m-1} \sigma \alpha \beta, & \omega \gamma \sigma & =\omega \nu \delta, \\
\varrho \omega \gamma & =\alpha \beta \gamma+\lambda(\alpha \beta \gamma \sigma)^{m-1} \alpha \beta \gamma, & \gamma \sigma \varrho & =\nu \delta \varrho, \\
(\alpha \beta \gamma \sigma)^{m} \alpha=0, & (\gamma \sigma \alpha \beta)^{m} \gamma & =0 .
\end{array}
$$

We call $S(m, \lambda)$ with $m \geq 2$ a higher spherical algebra. For $m=1$ and $\lambda \in K \backslash\{-1,0\}$, this is the non-singular spherical algebra $S(1+\lambda)$ investigated in [11, Section 3]. The above quiver is its Gabriel quiver, and $S(1+\lambda)$ is a surface algebra (in the sense of [11]) given by the following triangulation of the sphere $S^{2}$ in $\mathbb{R}^{3}$

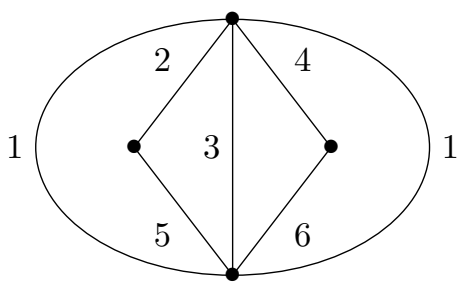

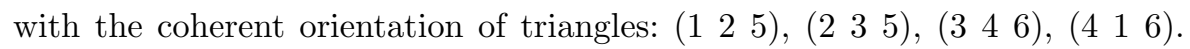
We note that the non-singular spherical algebras in [11] appear since in the general setting for weighted surface algebras we allow 'virtual' arrows.

The following theorem describes basic properties of higher spherical algebras.

Theorem 1. Let $S=S(m, \lambda)$ be a higher spherical algebra. Then the following statements hold:

(i) $S$ is a finite-dimensional algebra with $\operatorname{dim}_{K} S=36 m+4$.

(ii) $S$ is a symmetric algebra.

(iii) $S$ is a periodic algebra of period 4.

(iv) $S$ is a tame algebra of non-polynomial growth.

It follows from the above theorem that the higher spherical algebras $S(m, \lambda)$, $m \geq 2, \lambda \in K^{*}$, form an exotic family of algebras of generalized quaternion type (in the sense of [10]) whose Gabriel quiver is not 2-regular. The classification of the Morita equivalence classes of all algebras of generalized quaternion type with 2-regular Gabriel quivers having at least three vertices has been established in [10, Main Theorem]. During the work on this, surprisingly, we discovered new algebras, which we call higher tetrahedral algebras $\Lambda(m, \lambda)$, $m \geq 2, \lambda \in K^{*}$. They are introduced and studied in [9] (see Section 3 for definition and properties).

The following theorem relates these two classes of algebras.

Theorem 2. Let $m \geq 2$ be a natural number and $\lambda \in K^{*}$. Then the algebras $S(m, \lambda)$ and $\Lambda(m, \lambda)$ are derived equivalent. 
Then Theorem 1 is the consequence of Theorem 2, by applying general theory as described in Theorems 2.3, 2.4, 2.5 and 3.1.

The following problem arises naturally.

Problem. Describe the derived equivalence classes of higher tetrahedral algebras.

This seems to be a rather hard problem. We currently do not see new classes of tame symmetric periodic algebras, which are derived equivalent to higher tetrahedral (spherical) algebras.

For general background on the relevant representation theory, we refer to the books $[1,18,24,25]$.

2. Derived equivalences. In this section, we collect some facts on derived equivalences of algebras, which are needed in the proofs of Theorems 1 and 2 .

Let $A$ be an algebra over $K$. We denote by $D^{b}(\bmod A)$ the derived category of $\bmod A$, which is the localization of the homotopy category $K^{b}(\bmod A)$ of bounded complexes of modules from $\bmod A$ with respect to quasi-isomorphisms. Moreover, let $K^{b}\left(P_{A}\right)$ be the subcategory of $K^{b}(\bmod A)$ given by the complexes of projective modules in $\bmod A$. Two algebras $A$ and $B$ are called derived equivalent if the derived categories $D^{b}(\bmod A)$ and $D^{b}(\bmod B)$ are equivalent as triangulated categories. Following Rickard [21], a complex $T$ in $K^{b}\left(P_{A}\right)$ is called a tilting complex if the following properties are satisfied:

(1) $\operatorname{Hom}_{K^{b}\left(P_{A}\right)}(T, T[i])=0$ for all $i \neq 0$ in $\mathbb{Z}$,

(2) the full subcategory $\operatorname{add}(T)$ of $K^{b}\left(P_{A}\right)$ consisting of direct summands of direct sums of copies of $T$ generates $K^{b}\left(P_{A}\right)$ as a triangulated category.

Here, [] denotes the translation functor by shifting any complex one degree to the left.

The following theorem is due to Rickard [21, Theorem 6.4].

Theorem 2.1. Two algebras $A$ and $B$ are derived equivalent if and only if there is a tilting complex $T$ in $K^{b}\left(P_{A}\right)$ such that $B \cong \operatorname{End}_{K^{b}\left(P_{A}\right)}(T)$.

We will need the following special case of an alternating sum formula established by Happel [18, Sections III.1.3 and III.1.4].

Proposition 2.2. Let $A$ be an algebra and $Q=\left(Q_{r}\right)_{r \in \mathbb{Z}}, R=\left(R_{s}\right)_{s \in \mathbb{Z}}$ two complexes in $K^{b}\left(P_{A}\right)$ such that $\operatorname{Hom}_{K^{b}\left(P_{A}\right)}(Q, R[i])=0$ for any $i \neq 0$ in $\mathbb{Z}$. Then

$$
\operatorname{dim}_{K} \operatorname{Hom}_{K^{b}\left(P_{A}\right)}(Q, R)=\sum_{r, s}(-1)^{r-s} \operatorname{dim}_{K} \operatorname{Hom}_{A}\left(Q_{r}, R_{s}\right)
$$

We note that the right-hand side of the above formula can easily be computed using the Cartan matrix of $A$.

We end this section with the following collection of important results.

Theorem 2.3. Let $A$ and $B$ be derived equivalent algebras. Then $A$ is symmetric if and only if $B$ is symmetric.

Proof. This is [23, Corollary 5.3]. 
Theorem 2.4. Let $A$ and $B$ be derived equivalent algebras. Then $A$ is periodic if and only if $B$ is periodic. Moreover, if so, then they have the same period.

Proof. See [7, Theorem 2.9].

Theorem 2.5. Let $A$ and $B$ be derived equivalent self-injective algebras. Then the following equivalences hold:

(i) $A$ is tame if and only if $B$ is tame.

(ii) $A$ is of polynomial growth if and only if $B$ is of polynomial growth.

Proof. It follows from the assumption and [22, Corollary 2.2] (see also [23, Corollary 5.3]) that the algebras $A$ and $B$ are stably equivalent. Then the equivalences (i) and (ii) hold by [3, Theorems 4.4 and 5.6] and [19, Corollary 2].

3. Higher tetrahedral algebras. In this section, we recall some facts on higher tetrahedral algebras established in [9], which will be crucial in the proof of Theorem 2.

Consider the tetrahedron

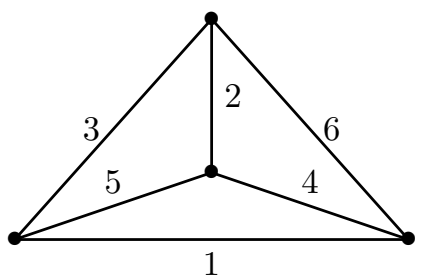

with the coherent orientation of triangles: (1 5 4), (2 53 ), (2 64 ), (1 63 ). Then, following [8, Section 6$]$, we have the associated triangulation quiver $(Q, f)$ of the form

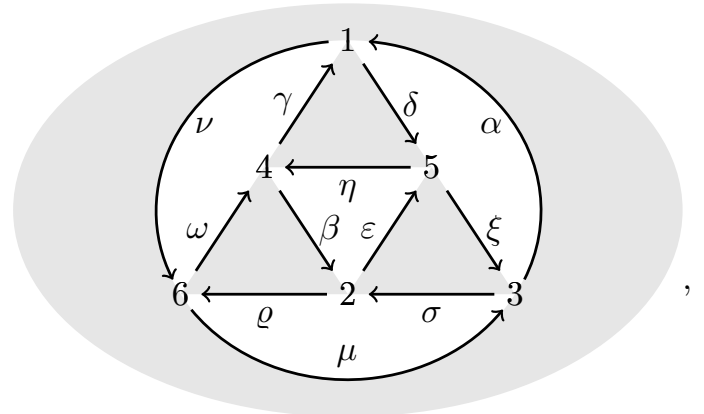

where $f$ is the permutation of arrows of order 3 described by the four shaded 3 -cycles. We denote by $g$ the permutation on the set of arrows of $Q$ whose $g$-orbits are the four white 3 -cycles.

Let $m \geq 2$ be a natural number and $\lambda \in K^{*}$. Following [9], the (nonsingular) tetrahedral algebra $\Lambda(m, \lambda)$ of degree $m$ is the algebra given by the above quiver $Q$ and the relations: 


$$
\begin{aligned}
& \gamma \delta=\beta \varepsilon+\lambda(\beta \varrho \omega)^{m-1} \beta \varepsilon, \quad \delta \eta=\nu \omega, \quad \eta \gamma=\xi \alpha, \quad \nu \mu=\delta \xi, \\
& \varrho \omega=\varepsilon \eta+\lambda(\varepsilon \xi \sigma)^{m-1} \varepsilon \eta, \quad \omega \beta=\mu \sigma, \quad \beta \varrho=\gamma \nu, \quad \mu \alpha=\omega \gamma, \\
& \xi \sigma=\eta \beta+\lambda(\eta \gamma \delta)^{m-1} \eta \beta, \quad \sigma \varepsilon=\alpha \delta, \quad \varepsilon \xi=\varrho \mu, \quad \alpha \nu=\sigma \varrho,
\end{aligned}
$$

$\left(\theta f(\theta) f^{2}(\theta)\right)^{m-1} \theta f(\theta) g(f(\theta))=0$ for any arrow $\theta$ in $Q$.

The following theorem is a consequence of $[9$, Theorems 1, 2, 3] and describes some basic properties of higher tetrahedral algebras.

Theorem 3.1. Let $\Lambda=\Lambda(m, \lambda)$ be a higher tetrahedral algebra with $m \geq 2$ and $\lambda \in K^{*}$. Then the following statements hold:

(i) $\Lambda$ is a finite-dimensional algebra with $\operatorname{dim}_{K} \Lambda=36 \mathrm{~m}$.

(ii) $\Lambda$ is a symmetric algebra.

(iii) $\Lambda$ is a periodic algebra of period 4 .

(iv) $\Lambda$ is a tame algebra of non-polynomial growth.

By counting the vectors in the basis of the higher tetrahedral algebra, as given after the proof of [9, Lemma 4.5], one obtains the following:

Proposition 3.2. Let $\Lambda=\Lambda(m, \lambda)$ be a higher tetrahedral algebra with $m \geq 2$ and $\lambda \in K^{*}$. Then the Cartan matrix $C_{\Lambda}$ of $\Lambda$ is of the form

$$
\left[\begin{array}{cccccc}
m+1 & m-1 & m & m & m & m \\
m-1 & m+1 & m & m & m & m \\
m & m & m+1 & m-1 & m & m \\
m & m & m-1 & m+1 & m & m \\
m & m & m & m & m+1 & m-1 \\
m & m & m & m & m-1 & m+1
\end{array}\right]
$$

4. Bases of higher spherical algebras. In this section, we describe bases and the Cartan matrices of higher spherical algebras.

Let $S=S(m, \lambda)$ be a higher spherical algebra with $m \geq 2$ and $\lambda \in K^{*}$. We start by collecting further identities in $S$, they follow directly from the relations defining $S$.

Lemma 4.1. The following relations hold in $S$ :

(i) $(\beta \gamma \sigma \alpha)^{m-1} \beta \gamma \sigma \varrho=0$ and $(\alpha \beta \gamma \sigma)^{m} \varrho=0$.

(ii) $\omega \gamma \sigma \alpha(\beta \gamma \sigma \alpha)^{m-1}=0$ and $\omega(\gamma \sigma \alpha \beta)^{m}=0$.

(iii) $(\sigma \alpha \beta \gamma)^{m-1} \sigma \alpha \beta \nu=0$ and $(\gamma \sigma \alpha \beta)^{m} \nu=0$.

(iv) $\delta \alpha \beta \gamma(\sigma \alpha \beta \gamma)^{m-1}=0$ and $\delta(\alpha \beta \gamma \sigma)^{m}=0$.

(v) $(\varrho \omega \nu \delta)^{r}=(\alpha \beta \gamma \sigma)^{r}$ and $(\nu \delta \varrho \omega)^{r}=(\gamma \sigma \alpha \beta)^{r}$, for $2 \leq r \leq m$.

For example, consider part (i). We have $\lambda(\beta \gamma \sigma \alpha)^{m-1} \beta \gamma \sigma=\beta \nu \delta-\beta \gamma \sigma$. We postmultiply this with $\rho$ and get zero since $\gamma \sigma \rho=\nu \delta \rho$. The second part follows by rewriting the first part and premultiply with $\alpha$. For part (v), starting with

$$
\rho \omega \nu \delta=\alpha \beta \gamma \sigma+\lambda(\alpha \beta \gamma \sigma)^{m}
$$

and squaring, one gets $(\rho \omega \nu \delta)^{2}=(\alpha \beta \gamma \sigma)^{2}$ and (v) follows by induction.

Using the relations, one may compute a basis for $S$. For convenience, we give a basis $\mathcal{B}_{i}$ for $e_{i} S$ explicitly, for each $i$. For $1 \leq i \leq 4$, we use the relevant 
rotation of $(\alpha \beta \gamma \sigma)^{r}$ and postmultiply by the shortest path to a vertex $j$ for $1 \leq j \leq 6$. Similarly, for $i=5,6$, we use the relevant rotation of $(\delta \rho \omega \nu)^{r}$.

Let $\mathcal{B}_{1}^{\prime}=\left\{e_{1}, \alpha, \alpha \beta, \alpha \beta \gamma, \rho \omega \nu, \rho\right\}$ and

$$
\mathcal{B}_{1}=\left\{(\alpha \beta \gamma \sigma)^{r} \varphi \mid 0 \leq r \leq m-1, \varphi \in \mathcal{B}_{1}^{\prime}\right\} \cup\left\{\rho \omega,(\alpha \beta \gamma \sigma)^{m}\right\} .
$$

Let $\mathcal{B}_{2}^{\prime}=\left\{\beta \gamma \sigma, e_{2}, \beta, \beta \gamma, \beta \nu\right\}$ and take

$$
\begin{aligned}
\mathcal{B}_{2}= & \left\{(\beta \gamma \sigma \alpha)^{r} \varphi \mid 0 \leq r \leq m-1, \varphi \in \mathcal{B}_{2}^{\prime}\right\} \\
& \cup\left\{(\beta \gamma \sigma \alpha)^{r} \beta \gamma \sigma \rho \mid 0 \leq r \leq m-2\right\} \cup\left\{(\beta \gamma \sigma \alpha)^{m}\right\} .
\end{aligned}
$$

Note that $(\beta \gamma \sigma \alpha)^{m-1} \beta \gamma \sigma \rho=0$ by $4.1(\mathrm{i})$. Let $\mathcal{B}_{3}^{\prime}=\left\{\gamma \sigma, \gamma \sigma \alpha, e_{3}, \gamma, \nu, \gamma \sigma \rho\right\}$ and

$$
\mathcal{B}_{3}=\left\{(\gamma \sigma \alpha \beta)^{r} \varphi \mid 0 \leq r \leq m-1, \varphi \in \mathcal{B}_{3}^{\prime}\right\} \cup\left\{\nu \delta,(\gamma \sigma \alpha \beta)^{m}\right\} .
$$

Let $\mathcal{B}_{4}^{\prime}=\left\{\sigma, \sigma \alpha, \sigma \alpha \beta, e_{4}, \sigma \rho\right\}$ and

$$
\begin{aligned}
\mathcal{B}_{4}= & \left\{(\sigma \alpha \beta \gamma)^{r} \varphi \mid 0 \leq r \leq m-1, \varphi \in \mathcal{B}_{4}^{\prime}\right\} \\
& \cup\left\{(\sigma \alpha \beta \gamma)^{r} \sigma \alpha \beta \nu \mid 0 \leq r \leq m-2\right\} \cup\left\{(\sigma \alpha \beta \gamma)^{m}\right\} .
\end{aligned}
$$

Note that $(\sigma \alpha \beta \gamma)^{m-1} \sigma \alpha \beta \nu=0$ by 4.1 (iii). For $\mathcal{B}_{5}$ and $\mathcal{B}_{6}$, first observe that $(\delta \rho \omega \nu)^{m-1} \delta \rho \omega \gamma=0$ and $(\omega \nu \delta \rho)^{m-1} \omega \nu \delta \alpha=0$ (these follow from Lemma 4.1 parts (ii), (iv), and (v)). Let $\mathcal{B}_{5}^{\prime}=\left\{\delta, \delta \alpha, \delta \rho \omega, e_{5}, \delta \rho\right\}$. Then take

$$
\begin{aligned}
\mathcal{B}_{5}= & \left\{(\delta \rho \omega \nu)^{r} \varphi \mid 0 \leq r \leq m-1, \varphi \in \mathcal{B}_{5}^{\prime}\right\} \\
& \cup\left\{(\delta \rho \omega \nu)^{r} \delta \rho \omega \gamma \mid 0 \leq r \leq m-2\right\} \cup\left\{(\delta \rho \omega \nu)^{m}\right\} .
\end{aligned}
$$

Let $\mathcal{B}_{6}^{\prime}=\left\{\omega \nu \delta, \omega, \omega \gamma, \omega \nu, e_{6}\right\}$. Take

$$
\begin{aligned}
\mathcal{B}_{6}= & \left\{(\omega \nu \delta \rho)^{r} \varphi \mid 0 \leq r \leq m-1, \varphi \in \mathcal{B}_{6}^{\prime}\right\} \\
& \cup\left\{(\omega \nu \delta \rho)^{r} \omega \nu \delta \alpha \mid 0 \leq r \leq m-2\right\} \cup\left\{(\omega \nu \delta \rho)^{m}\right\} .
\end{aligned}
$$

From this description, one sees directly that the size of $\mathcal{B}_{i} e_{j}$ is equal to the $i j$-th entry of the Cartan matrix below.

Proposition 4.2. The Cartan matrix $C_{S}$ of $S$ is of the form

$$
\left[\begin{array}{cccccc}
m+1 & m & m+1 & m & m & m \\
m & m+1 & m & m & m & m-1 \\
m+1 & m & m+1 & m & m & m \\
m & m & m & m+1 & m-1 & m \\
m & m & m & m-1 & m+1 & m \\
m & m-1 & m & m & m & m+1
\end{array}\right]
$$

In particular, $\operatorname{dim}_{K} S=36 m+4$.

5. Proof of Theorem 2. Let $\Lambda=\Lambda(m, \lambda)$ for some fixed $m \geq 2$ and $\lambda \in K^{*}$. For each vertex $i$ of the quiver $Q$ defining $\Lambda$, we denote by $P_{i}=e_{i} \Lambda$ the associated indecomposable projective module in $\bmod \Lambda$. Moreover, for any arrow $\theta$ from 
$j$ to $k$ in $Q$, we denote by $\theta: P_{k} \rightarrow P_{j}$ the homomorphism in $\bmod \Lambda$ given by the left multiplication by $\theta$. We consider the following complexes in $K^{b}\left(P_{\Lambda}\right)$ :

$$
\begin{array}{ll}
T_{1}: & 0 \longrightarrow P_{1} \longrightarrow 0, \\
T_{2}: & 0 \longrightarrow P_{5} \longrightarrow 0, \\
T_{4}: & 0 \longrightarrow P_{3} \longrightarrow 0, \\
T_{5}: & 0 \longrightarrow P_{4} \longrightarrow 0, \\
T_{6}: & 0 \longrightarrow P_{6} \longrightarrow 0,
\end{array}
$$

concentrated in degree 0 , and

$$
T_{3}: \quad 0 \longrightarrow P_{2} \stackrel{\left[\begin{array}{c}
-\sigma \\
\beta
\end{array}\right]}{\longrightarrow} P_{3} \oplus P_{4} \longrightarrow 0,
$$

concentrated in degrees 1 and 0 . Moreover, we set

$$
T=T_{1} \oplus T_{2} \oplus T_{3} \oplus T_{4} \oplus T_{5} \oplus T_{6} .
$$

Lemma 5.1. $T$ is a tilting complex in $K^{b}\left(P_{\Lambda}\right)$.

Proof. We show that $T$ satisfies the conditions (1) and (2) defining a tilting complex.

(1) Since the summands $T_{i}$ of $T$ different from $T_{3}$ are concentrated in degree 0 , it is sufficient to show the equalities

$$
\operatorname{Hom}_{K^{b}\left(P_{\Lambda}\right)}\left(T_{3}, T_{r}[1]\right)=0, \quad \operatorname{Hom}_{K^{b}\left(P_{\Lambda}\right)}\left(T_{r}, T_{3}[-1]\right)=0,
$$

for $r \in\{1,2, \ldots, 6\}$. The first equalities hold because, for any $i \in\{1,3,4$, $5,6\}$, we have $e_{i} \Lambda e_{2} \subseteq \Lambda e_{3} \Lambda e_{2}+\Lambda e_{4} \Lambda e_{2}=\Lambda \sigma+\Lambda \beta$, and hence any non-zero homomorphism $f: P_{2} \rightarrow P_{i}$ with $i \neq 2$ factors through $\left[\begin{array}{c}-\sigma \\ \beta\end{array}\right]$ : $P_{2} \rightarrow P_{3} \oplus P_{4}$. For the proof that the second equalities hold, it is enough to show that for any non-zero homomorphism $g: P_{i} \rightarrow P_{2}$ with $i \neq 2$, the composition $\left[\begin{array}{c}-\sigma \\ \beta\end{array}\right] g$ is non-zero. We fix $i \in\{1,3,4,5,6\}$. Let $u$ be a non-zero path in $\Lambda$ from 2 to $i$, say of length $k$. Assume first that $i$ is different from 3 and 4 . Then it follows from [9, Lemmas 4.4 and 4.5] that $1 \leq k \leq 3 m-2$ and hence both paths $\sigma u$ and $\beta u$ are of lengths $\leq 3 m-1$, and are non-zero. Assume now that $i$ is equal to 3 or 4 , say $i=3$. By $[9$, Lemmas 4.4 and 4.5] again, the longest non-zero path in $\Lambda$ from vertex 2 to vertex 3 has length $3 m-1$, hence $k \leq 3 m-1$, and $\beta u$ is a non-zero path of length at most $3 m$ ending at vertex 3 .

(2) We consider the complex in $K^{b}\left(P_{\Lambda}\right)$

$$
T_{3}^{\prime}: \quad 0 \longrightarrow P_{2} \longrightarrow 0,
$$

concentrated in degree 1 . Moreover, let $f: T_{4} \oplus T_{5} \rightarrow T_{3}$ be the morphism in $K^{b}\left(P_{\Lambda}\right)$ given by the identity homomorphism $\operatorname{id}_{P_{3} \oplus P_{4}}: P_{3} \oplus P_{4} \rightarrow$ $P_{3} \oplus P_{4}$ in $\bmod \Lambda$. Then we have in $K^{b}\left(P_{\Lambda}\right)$ the standard triangle

$$
T_{4} \oplus T_{5} \stackrel{f}{\longrightarrow} T_{3} \stackrel{\alpha(f)}{\longrightarrow} C(f) \stackrel{\beta(f)}{\longrightarrow}\left(T_{4} \oplus T_{5}\right)[1],
$$


where $C(f)$ is the mapping cone of $f$. Since $C(f)$ and $T_{3}^{\prime}$ are isomorphic in $K^{b}\left(P_{\Lambda}\right)$, we conclude that add $(T)$ generates the triangulated category $K^{b}\left(P_{\Lambda}\right)$.

We define $R=R(m, \lambda)=\operatorname{End}_{K^{b}\left(P_{\Lambda}\right)}(T)$. Then the $\tilde{P}_{i}=\operatorname{Hom}_{K^{b}\left(P_{\Lambda}\right)}\left(T, T_{i}\right)$, $i \in\{1,2, \ldots, 6\}$, form a complete family of pairwise non-isomorphic indecomposable projective modules in $\bmod R$. We abbreviate $S=S(m, \lambda)$, and use the ordering $\bar{P}_{i}=e_{i} S, i \in\{1,2, \ldots, 6\}$, of the indecomposable projective modules in $\bmod S$ corresponding to the numbering of vertices of the quiver $\Delta$ defining $S$. With this notation, we have the following.

Lemma 5.2. The Cartan matrices $C_{R}$ and $C_{S}$ coincide. In particular, the algebras $R$ and $S$ have both dimension $36 m+4$.

Proof. This follows from computing $\operatorname{dim}_{K} \operatorname{Hom}_{K^{b}\left(P_{\Lambda}\right)}\left(T_{i}, T_{j}\right), i, j \in\{1,2$, $\ldots, 6\}$, using Proposition 2.2, and the shape of the Cartan matrix $C_{\Lambda}$ of $\Lambda$ given in Proposition 3.2.

We now define irreducible morphisms between the summands of $T$ in $K^{b}\left(P_{\Lambda}\right)$ :

$$
\begin{aligned}
& \tilde{\alpha}: T_{2} \rightarrow T_{1} \text {, given by } \delta: P_{5} \rightarrow P_{1}, \\
& \tilde{\beta}: T_{3} \rightarrow T_{2} \text {, given by }\left[\xi \quad \eta+\lambda(\eta \gamma \delta)^{m-1} \eta\right]: P_{3} \oplus P_{4} \rightarrow P_{5} \text {, } \\
& \tilde{\gamma}: T_{4} \rightarrow T_{3} \text {, given by }\left[\begin{array}{l}
1 \\
0
\end{array}\right]: P_{3} \rightarrow P_{3} \oplus P_{4} \text {, } \\
& \tilde{\sigma}: T_{1} \rightarrow T_{4} \text {, given by } \alpha: P_{1} \rightarrow P_{3} \text {, } \\
& \tilde{\delta}: T_{1} \rightarrow T_{5} \text {, given by } \gamma: P_{1} \rightarrow P_{4} \text {, } \\
& \tilde{\varrho}: T_{6} \rightarrow T_{1} \text {, given by } \nu: P_{6} \rightarrow P_{1} \text {, } \\
& \tilde{\omega}: T_{3} \rightarrow T_{6} \text {, given by }\left[\begin{array}{ll}
\mu & \omega
\end{array}\right]: P_{3} \oplus P_{4} \rightarrow P_{6} \text {, } \\
& \tilde{\nu}: T_{5} \rightarrow T_{3} \text {, given by }\left[\begin{array}{l}
0 \\
1
\end{array}\right]: P_{4} \rightarrow P_{3} \oplus P_{4} \text {. }
\end{aligned}
$$

We then obtain the irreducible homomorphisms between the indecomposable projective modules in $\bmod R$ :

$$
\begin{array}{ll}
\alpha=\operatorname{Hom}_{K^{b}\left(P_{\Lambda}\right)}(T, \tilde{\alpha}): \tilde{P}_{2} \rightarrow \tilde{P}_{1}, & \beta=\operatorname{Hom}_{K^{b}\left(P_{\Lambda}\right)}(T, \tilde{\beta}): \tilde{P}_{3} \rightarrow \tilde{P}_{2}, \\
\gamma=\operatorname{Hom}_{K^{b}\left(P_{\Lambda}\right)}(T, \tilde{\gamma}): \tilde{P}_{4} \rightarrow \tilde{P}_{3}, & \sigma=\operatorname{Hom}_{K^{b}\left(P_{\Lambda}\right)}(T, \tilde{\sigma}): \tilde{P}_{1} \rightarrow \tilde{P}_{4}, \\
\delta=\operatorname{Hom}_{K^{b}\left(P_{\Lambda}\right)}(T, \tilde{\delta}): \tilde{P}_{1} \rightarrow \tilde{P}_{4}, & \varrho=\operatorname{Hom}_{K^{b}\left(P_{\Lambda}\right)}(T, \tilde{\varrho}): \tilde{P}_{6} \rightarrow \tilde{P}_{1}, \\
\omega=\operatorname{Hom}_{K^{b}\left(P_{\Lambda}\right)}(T, \tilde{\omega}): \tilde{P}_{3} \rightarrow \tilde{P}_{6}, & \nu=\operatorname{Hom}_{K^{b}\left(P_{\Lambda}\right)}(T, \tilde{\nu}): \tilde{P}_{5} \rightarrow \tilde{P}_{3},
\end{array}
$$

which are representatives of all irreducible homomorphisms between the modules $\tilde{P}_{i}, i \in\{1,2, \ldots, 6\}$, in $\bmod R$. This shows that the Gabriel quiver $Q_{R}$ of $R$ is the quiver $\Delta$ defining the algebra $S$.

Theorem 5.3. The algebras $R$ and $S$ are isomorphic. 
Proof. We first prove that the following identities hold in $R$ :

$$
\begin{aligned}
\alpha \beta \gamma & =\varrho \omega \gamma, \\
\sigma \alpha \beta & =\sigma \varrho \omega, \\
\gamma \sigma \varrho & =\nu \delta \varrho, \\
\omega \gamma \sigma & =\omega \nu \delta, \\
\beta \nu \delta & =\beta \gamma \sigma+\lambda(\beta \gamma \sigma \alpha)^{m-1} \beta \gamma \sigma, \\
\nu \delta \alpha & =\gamma \sigma \alpha+\lambda(\gamma \sigma \alpha \beta)^{m-1} \gamma \sigma \alpha, \\
\delta \alpha \beta & =\delta \varrho \omega+\lambda(\delta \varrho \omega \nu)^{m-1} \delta \varrho \omega, \\
\alpha \beta \nu & =\varrho \omega \nu+\lambda(\varrho \omega \nu \delta)^{m-1} \varrho \omega \nu, \\
(\alpha \beta \gamma \sigma)^{m} \alpha & =0 \\
(\gamma \sigma \alpha \beta)^{m} \gamma & =0 .
\end{aligned}
$$

For (1), it is enough to show that $\tilde{\alpha} \tilde{\beta} \tilde{\gamma}=\tilde{\varrho} \tilde{\omega} \tilde{\gamma}$. We have $\tilde{\alpha} \tilde{\beta} \tilde{\gamma}=\delta \xi: P_{3} \rightarrow P_{1}$ and $\tilde{\varrho} \tilde{\omega} \tilde{\gamma}=\nu \mu: P_{3} \rightarrow P_{1}$, with $\delta \xi=\nu \mu$ in $\Lambda$, and so the required equality holds.

For (2), it is enough to show that $\tilde{\sigma} \tilde{\alpha} \tilde{\beta}=\tilde{\sigma} \varrho \tilde{\varrho} \tilde{\omega}$. We have $\tilde{\sigma} \tilde{\alpha} \tilde{\beta}=[\alpha \delta \xi \quad \alpha \delta \eta+$ $\left.\lambda \alpha \delta(\eta \gamma \delta)^{m-1} \eta\right]: P_{3} \oplus P_{4} \rightarrow P_{3}$ and $\tilde{\sigma} \tilde{\varrho} \tilde{\omega}=[\alpha \nu \mu, \alpha \nu \omega]: P_{3} \oplus P_{4} \rightarrow P_{3}$. Moreover, we have in $\Lambda$ the equalities

$$
\alpha \nu \omega=\sigma \varrho \omega=\sigma \varepsilon \eta+\lambda \sigma(\varepsilon \xi \sigma)^{m-1} \varepsilon \eta=\alpha \delta \eta+\lambda \alpha \delta(\xi \sigma \varepsilon)^{m-1} \eta .
$$

Hence the required equality holds.

For $(3)$, we prove that $\tilde{\nu} \tilde{\delta} \tilde{\varrho}-\tilde{\gamma} \tilde{\sigma} \tilde{\varrho}=0$ in $K^{b}\left(P_{\Lambda}\right)$. We have

$$
\tilde{\gamma} \tilde{\sigma} \tilde{\varrho}=\left[\begin{array}{c}
\alpha \nu \\
0
\end{array}\right]: P_{6} \rightarrow P_{3} \oplus P_{4} \quad \text { and } \quad \tilde{\nu} \tilde{\delta} \tilde{\varrho}=\left[\begin{array}{c}
0 \\
\gamma \nu
\end{array}\right]: P_{6} \rightarrow P_{3} \oplus P_{4} .
$$

Moreover, we have the following commutative diagram in $\bmod \Lambda$

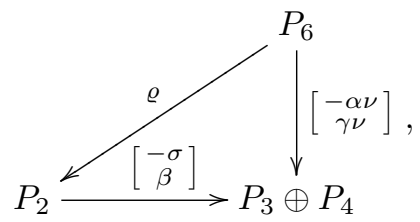

because $\alpha \nu=\sigma \varrho$ and $\gamma \nu=\beta \varrho$ in $\Lambda$. This proves the claim.

For (4), we note that $\tilde{\omega} \tilde{\gamma} \tilde{\sigma}=\mu \alpha: P_{1} \rightarrow P_{6}$ and $\tilde{\omega} \tilde{\nu} \tilde{\delta}=\omega \gamma: P_{1} \rightarrow P_{6}$, with $\mu \alpha=\omega \gamma$.

For $(5)$, we prove the equality $\tilde{\beta} \tilde{\nu} \tilde{\delta}=\tilde{\beta} \tilde{\gamma} \tilde{\sigma}+\lambda(\tilde{\beta} \tilde{\gamma} \tilde{\sigma} \tilde{\alpha})^{m-1} \tilde{\beta} \tilde{\gamma} \tilde{\sigma}$. Observe that

$$
\begin{gathered}
\tilde{\beta} \tilde{\nu} \tilde{\delta}=\eta \gamma+\lambda(\eta \gamma \delta)^{m-1} \eta \gamma: P_{1} \rightarrow P_{5}, \\
\tilde{\beta} \tilde{\gamma} \tilde{\sigma}=\xi \alpha=\eta \gamma: P_{1} \rightarrow P_{5}, \\
\lambda(\tilde{\beta} \tilde{\gamma} \tilde{\sigma} \tilde{\alpha})^{m-1} \tilde{\beta} \tilde{\gamma} \tilde{\sigma}=\lambda(\xi \alpha \delta)^{m-1} \eta \gamma=\lambda(\eta \gamma \delta)^{m-1} \eta \gamma: P_{1} \rightarrow P_{5},
\end{gathered}
$$

and hence the required equality holds. 
For (6), we prove that $\tilde{\nu} \tilde{\delta} \tilde{\alpha}=\tilde{\gamma} \tilde{\sigma} \tilde{\alpha}+\lambda(\tilde{\gamma} \tilde{\sigma} \tilde{\alpha} \tilde{\beta})^{m-1} \tilde{\gamma} \tilde{\sigma} \tilde{\alpha}$ in $K^{b}\left(P_{\Lambda}\right)$. We first observe that

$$
\begin{aligned}
\tilde{\nu} \tilde{\delta} \tilde{\alpha} & =\left[\begin{array}{c}
0 \\
\gamma \delta
\end{array}\right]=\left[\begin{array}{c}
0 \\
\beta \varepsilon+\lambda(\beta \varrho \omega)^{m-1} \beta \varepsilon
\end{array}\right]: P_{5} \rightarrow P_{3} \oplus P_{4}, \\
\tilde{\gamma} \tilde{\sigma} \tilde{\alpha} & =\left[\begin{array}{c}
\alpha \delta \\
0
\end{array}\right]: P_{5} \rightarrow P_{3} \oplus P_{4}, \\
\tilde{\gamma} \tilde{\sigma} \tilde{\alpha} \tilde{\beta} & =\left[\begin{array}{c}
\alpha \delta \xi \alpha \delta \eta+\lambda \alpha \delta(\eta \gamma \delta)^{m-1} \eta \\
0
\end{array}\right]: P_{3} \oplus P_{4} \rightarrow P_{3} \oplus P_{4} .
\end{aligned}
$$

Moreover, $\alpha \delta=\sigma \varepsilon$, and hence

$$
\lambda(\alpha \delta \xi)^{m-1} \alpha \delta=\lambda(\sigma \varepsilon \xi)^{m-1} \sigma \varepsilon=\lambda \sigma(\varepsilon \xi \sigma)^{m-1} \varepsilon .
$$

But then

$$
\tilde{\gamma} \tilde{\sigma} \tilde{\alpha}+\lambda(\tilde{\gamma} \tilde{\sigma} \tilde{\alpha} \tilde{\beta})^{m-1} \tilde{\gamma} \tilde{\sigma} \tilde{\alpha}=\left[\begin{array}{c}
\sigma \varepsilon+\lambda \sigma(\varepsilon \xi \sigma)^{m-1} \varepsilon \\
0
\end{array}\right]: P_{5} \rightarrow P_{3} \oplus P_{4} .
$$

Further, we have the following commutative diagram in $\bmod \Lambda$

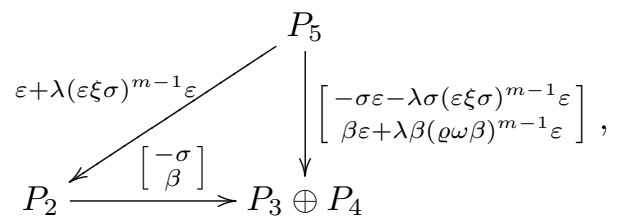

because $\varrho \omega \beta=\varrho \mu \sigma=\varepsilon \xi \sigma$ in $\Lambda$. This shows that $\tilde{\nu} \tilde{\delta} \tilde{\alpha}-\tilde{\gamma} \tilde{\sigma} \tilde{\alpha}-\lambda(\tilde{\gamma} \tilde{\sigma} \tilde{\alpha} \tilde{\beta})^{m-1} \tilde{\gamma} \tilde{\sigma} \tilde{\alpha}$ $=0$ in $K^{b}\left(P_{\Lambda}\right)$, and hence the required equality holds.

For $(7)$, we prove that $\tilde{\delta} \tilde{\alpha} \tilde{\beta}=\tilde{\delta} \tilde{\varrho} \tilde{\omega}+\lambda(\tilde{\delta} \tilde{\varrho} \tilde{\omega} \tilde{\nu})^{m-1} \tilde{\delta} \tilde{\varrho} \tilde{\omega}$. We have

$$
\begin{aligned}
& \tilde{\delta} \tilde{\alpha} \tilde{\beta}=\left[\begin{array}{ll}
\gamma \delta \xi & \gamma \delta \eta+\lambda \gamma \delta(\eta \gamma \delta)^{m-1} \eta
\end{array}\right]: P_{3} \oplus P_{4} \rightarrow P_{4}, \\
& \tilde{\delta} \tilde{\varrho} \tilde{\omega}=\left[\begin{array}{ll}
\gamma \nu \mu & \gamma \nu \omega
\end{array}\right]: P_{3} \oplus P_{4} \rightarrow P_{4},
\end{aligned}
$$

and $\delta \xi=\nu \mu, \delta \eta=\nu \omega$ in $\Lambda$. Furthermore,

$$
\tilde{\delta} \tilde{\varrho} \tilde{\omega} \tilde{\nu}=\gamma \nu \omega=\gamma \delta \eta: P_{4} \rightarrow P_{4} .
$$

Hence we obtain

$$
\lambda(\tilde{\delta} \tilde{\varrho} \tilde{\omega} \tilde{\nu})^{m-1} \tilde{\delta} \tilde{\varrho} \tilde{\omega}=\left[\lambda(\gamma \delta \eta)^{m-1} \gamma \delta \xi \quad \lambda(\gamma \delta \eta)^{m-1} \gamma \delta \eta\right]: P_{3} \oplus P_{4} \rightarrow P_{4} .
$$

We note that

$$
\lambda(\gamma \delta \eta)^{m-1} \gamma \delta \xi=\lambda\left(\gamma f(\gamma) f^{2}(\gamma)\right)^{m-1} \gamma f(\gamma) g(f(\gamma))=0
$$

Therefore, the required equality holds.

For (8), we have to show that $\tilde{\alpha} \tilde{\beta} \tilde{\nu}=\tilde{\varrho} \tilde{\omega} \tilde{\nu}+\lambda(\tilde{\varrho} \tilde{\omega} \tilde{\nu} \tilde{\delta})^{m-1} \tilde{\varrho} \tilde{\omega} \tilde{\nu}$. We have

$$
\begin{aligned}
\tilde{\alpha} \tilde{\beta} \tilde{\nu} & =\delta \eta+\lambda \delta(\eta \gamma \delta)^{m-1} \eta=\delta \eta+\lambda(\delta \eta \gamma)^{m-1} \delta \eta: P_{4} \rightarrow P_{1}, \\
\tilde{\varrho} \tilde{\omega} \tilde{\nu} & =\nu \omega=\delta \eta: P_{4} \rightarrow P_{1}, \\
\tilde{\varrho} \tilde{\omega} \tilde{\nu} \tilde{\delta} & =\nu \omega \gamma=\delta \eta \gamma: P_{1} \rightarrow P_{1},
\end{aligned}
$$

and then

$$
\lambda(\tilde{\varrho} \tilde{\omega} \tilde{\nu} \tilde{\delta})^{m-1} \tilde{\varrho} \tilde{\omega} \tilde{\nu}=\lambda(\delta \eta \gamma)^{m-1} \delta \eta: P_{4} \rightarrow P_{1} .
$$

Hence the required equality holds. 
For (9), we observe that

$$
(\tilde{\alpha} \tilde{\beta} \tilde{\gamma} \tilde{\sigma})^{m} \tilde{\alpha}=(\delta \xi \alpha)^{m} \delta: P_{5} \rightarrow P_{1},
$$

and this is zero, because in $\Lambda$, the element $(\delta \xi \alpha)^{m}$ belongs to the socle of $P_{1}$ (see [9, Lemma 4.2]).

For (10), we observe that $\tilde{\sigma} \tilde{\alpha} \tilde{\beta} \tilde{\gamma}=\alpha \delta \xi: P_{3} \rightarrow P_{3}$, and therefore

$$
(\tilde{\gamma} \tilde{\sigma} \tilde{\alpha} \tilde{\beta})^{m} \tilde{\gamma}=\left[\begin{array}{c}
(\alpha \delta \xi)^{m} \\
0
\end{array}\right]=\left[\begin{array}{c}
(\sigma \varepsilon \xi)^{m} \\
0
\end{array}\right]: P_{3} \rightarrow P_{3} \oplus P_{4}
$$

Then, we have in $\bmod \Lambda$ the commutative diagram

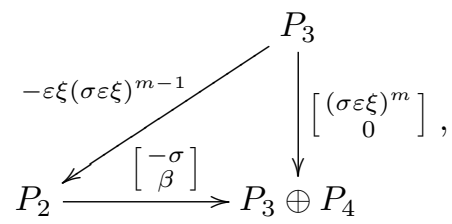

because we have in $\Lambda$ the qualities

$$
\beta \varepsilon \xi(\sigma \varepsilon \xi)^{m-1}=\beta \varrho \omega(\sigma \varepsilon \xi)^{m-1}=\cdots=(\beta \varrho \omega)^{m-1} \beta \varepsilon \xi=(\beta \varrho \omega)^{m-1} \beta \varrho \mu=0 .
$$

Therefore, $(\tilde{\gamma} \tilde{\sigma} \tilde{\alpha} \tilde{\beta})^{m} \tilde{\gamma}=0$ in $K^{b}\left(P_{\Lambda}\right)$, and the Equality (10) holds.

We also observe that, in $R$, we have by (1) and (4) that $\alpha \beta \gamma \sigma=\varrho \omega \gamma \sigma=$ $\varrho \omega \nu \delta$.

To obtain the defining relations for $S$, we replace $\varrho$ by $\varrho^{*}=\varrho+\lambda(\alpha \beta \gamma \sigma)^{m-1}$ $\varrho=\varrho+\lambda(\varrho \omega \nu \delta)^{m-1} \varrho$. Then Identities (1), (2), (3), (7), (8) are replaced by the following identities:

$$
\begin{gathered}
\varrho^{*} \omega \gamma=\varrho \omega \gamma+\lambda(\alpha \beta \gamma \sigma)^{m-1} \varrho \omega \gamma=\alpha \beta \gamma+\lambda(\alpha \beta \gamma \sigma)^{m-1} \alpha \beta \gamma, \\
\sigma \varrho^{*} \omega=\sigma \varrho \omega+\lambda \sigma(\alpha \beta \gamma \sigma)^{m-1} \varrho \omega=\sigma \alpha \beta+\lambda(\sigma \alpha \beta \gamma)^{m-1} \sigma \alpha \beta, \\
\gamma \sigma \varrho^{*}=\gamma \sigma \varrho+\lambda\left(\gamma \sigma(\varrho \omega \nu \delta)^{m-1} \varrho\right)=\gamma \sigma \varrho+\lambda(\gamma \sigma \varrho)(\omega \nu \sigma \varrho)^{m-1} \\
=\nu \delta \varrho+\lambda\left(\nu \delta(\varrho \omega \nu \delta)^{m-1} \varrho\right)=\nu \delta \varrho+\lambda(\nu \delta \varrho)(\omega \nu \sigma \varrho)^{m-1}=\nu \delta \varrho^{*}, \\
\delta \varrho^{*} \omega=\delta\left(\varrho+\lambda(\varrho \omega \nu \delta)^{m-1} \varrho\right) \omega=\delta \varrho \omega+\lambda \delta(\varrho \omega \nu \delta)^{m-1} \varrho \omega \\
=\delta \varrho \omega+\lambda(\delta \varrho \omega \nu)^{m-1} \delta \varrho \omega=\delta \alpha \beta \\
\varrho^{*} \omega \nu=\left(\varrho+\lambda(\varrho \omega \nu \delta)^{m-1} \varrho\right) \omega \nu=\varrho \omega \nu+\lambda(\varrho \omega \nu \delta)^{m-1} \varrho \omega \nu=\alpha \beta \nu .
\end{gathered}
$$

Therefore, after replacing $\varrho$ by $\varrho^{*}$, the relations defining the algebra $S=$ $S(m, \lambda)$ are satisfied. Then, applying Lemma 5.2, we conclude that the algebras $R$ and $S$ are isomorphic.

Summing up, Theorem 2 follows from Theorems 2.1 and 5.3. 
Open Access. This article is distributed under the terms of the Creative Commons Attribution 4.0 International License (http://creativecommons.org/licenses/ by/4.0/), which permits unrestricted use, distribution, and reproduction in any medium, provided you give appropriate credit to the original author(s) and the source, provide a link to the Creative Commons license, and indicate if changes were made.

Publisher's Note Springer Nature remains neutral with regard to jurisdictional claims in published maps and institutional affiliations.

\section{References}

[1] Assem, I., Simson, D., Skowroński, A.: Elements of the representation theory of associative algebras 1: techniques of representation theory. In: London Mathematical Society Student Texts, vol. 65, pp. x+458. Cambridge University Press, Cambridge (2006)

[2] Białkowski, J., Erdmann, K., Skowroński, A.: Periodicity of self-injective algebras of polynomial growth. J. Algebra 443, 200-269 (2015)

[3] Crawley-Boevey, W.: Tame algebras and generic modules. Proc. Lond. Math. Soc. 63, 241-265 (1991)

[4] Derksen, H., Weyman, J., Zelevinsky, A.: Quivers with potentials and their representations. I. Mutations. Selecta Math. (N.S.) 14, 59-119 (2008)

[5] Derksen, H., Weyman, J., Zelevinsky, A.: Quivers with potentials and their representations. II. Applications to cluster algebras. J. Am. Math. Soc. 23, 749-790 (2010)

[6] Dugas, A.: Periodic resolutions and self-injective algebras of finite type. J. Pure Appl. Algebra 214, 990-1000 (2010)

[7] Erdmann, K., Skowroński, A.: Periodic algebras. In: Trends in Representation Theory of Algebras and Related Topics. European Mathematical Society Series Congress Reports, pp. 201-251. European Mathematical Society, Zürich (2008)

[8] Erdmann, K., Skowroński, A.: Weighted surface algebras. J. Algebra 505, 490558 (2018)

[9] Erdmann, K., Skowroński, A.: Higher tetrahedral algebras. Algebr. Represent. Theory 22, 387-406 (2019)

[10] Erdmann, K., Skowroński, A.: Algebras of generalized quaternion type. Adv. Math. 349, 1036-1116 (2019)

[11] Erdmann, K., Skowroński, A.: Weighted surface algebras: general version. arXiv:1902.04063

[12] Erdmann, K., Skowroński, A.: From Brauer graph algebras to biserial weighted surface algebras. J. Algebr. Comb. (2018). https://doi.org/10.1007/ s10801-018-0867-6

[13] Erdmann, K., Skowroński, A.: Algebras of generalized dihedral type. Nagoya Math. J. (2019). https://doi.org/10.1017/nmj.2019.1

[14] Fomin, S., Shapiro, M., Thurston, D.: Cluster algebras and triangulated surfaces. I. Cluster complexes. Acta Math. 201, 83-146 (2008) 
[15] Fock, V., Goncharov, A.: Moduli spaces of local systems and higher Teichmüller theory. Publ. Math. Inst. Hautes Études Sci. 103, 1-211 (2006)

[16] Geiss, C., Labardini-Fragoso, D., Schröer, J.: The representation type of Jacobian algebras. Adv. Math. 290, 364-452 (2016)

[17] Gekhtman, M., Shapiro, M., Vainshtein, A.: Cluster algebras and Poisson geometry. Mosc. Math. J. 3, 899-934 (2003)

[18] Happel, D.: Triangulated categories in the representation theory of finitedimensional algebras. In: London Mathematical Society Lecture Note Series, vol. 119, p. x+208. Cambridge University Press, Cambridge (1988)

[19] Krause, H., Zwara, G.: Stable equivalence and generic modules. Bull. Lond. Math. Soc. 32, 615-618 (2000)

[20] Labardini-Fragoso, D.: Quivers with potentials associated to triangulated surfaces. Proc. Lond. Math. Soc. 98, 797-839 (2009)

[21] Rickard, J.: Morita theory for derived categories. J. Lond. Math. Soc. 39, 436456 (1989)

[22] Rickard, J.: Derived categories and stable equivalence. J. Pure Appl. Algebra 61, 303-317 (1989)

[23] Rickard, J.: Derived equivalences as derived functors. J. Lond. Math. Soc. 43, 37-48 (1991)

[24] Simson, D., Skowroński, A.: Elements of the representation theory of associative algebras 3: representation-infinite tilted algebras. In: London Mathematical Society Student Texts, vol. 72. Cambridge University Press, Cambridge (2007)

[25] Skowroński, A., Yamagata, K.: Frobenius algebras I. Basic representation theory. In: European Mathematical Society Textbooks in Mathematics, p. xii+650. European Mathematical Society Publishing House, Zürich (2011)

KARIN ERDMANN

Mathematical Institute

Oxford University

ROQ

Oxford OX2 6GG

UK

e-mail: erdmann@maths.ox.ac.uk 


\author{
ANDRZEJ SKowroński \\ Faculty of Mathematics and Computer Science \\ Nicolaus Copernicus University \\ Chopina 12/18 \\ 87-100 Toruń \\ Poland \\ e-mail: skowron@mat.uni.torun.pl
}

Received: 11 May 2019 